\title{
Relação entre domínios de esquemas desconexão/rejeição e autonomia/desempenho prejudicados e dimensões clínicas de personalidade
}

\author{
Natany de Souza Batista Medeiros $\oplus^{1}$ \\ Bruno Gonçalves de Medeiros ${ }^{1}{ }^{1}$ \\ Victor Hugo Dias Pereira ${ }^{2}{ }^{2}$ \\ Ismael Ferreira da Costa $\odot^{2}$ \\ Melyssa Kellyane Cavalcanti Galdino $\odot^{2}$ \\ ${ }^{1}$ Universidade Federal do Rio Grande do Norte, RN, Brasil. \\ ${ }^{2}$ Universidade Federal da Paraíba, PB, Brasil.
}

\begin{abstract}
Resumo
Esquemas Iniciais Desadaptativos (EIDs) são padrões de respostas emocionais e cognitivas desenvolvidos pela interação entre personalidade e experiências na infância. O objetivo desse estudo foi investigar as relações entre os EIDs e as dimensões clínicas de personalidade em uma amostra não clínica. Para isso, 189 universitários responderam ao YSQ-S2, IDCP-2, BAI e BDI. Domínios de esquemas se correlacionaram com todas as dimensões clínicas de personalidade. Os dados sugerem que indivíduos com as dimensões Dependência, Instabilidade de Humor e Evitação a Críticas são mais suscetíveis a apresentarem esquemas dos domínios Desconexão/Rejeição e Autonomia e Desempenho Prejudicado. O trabalho permite a integração do estudo da Personalidade e da Terapia dos Esquemas.
\end{abstract}

Palavras-chave: dimensões clínicas de personalidade, esquemas iniciais desadaptativos, vulnerabilidade cognitiva.

\section{Relation between the schema domains of disconnection/rejection and autonomy/impaired performance and clinical dimensions of personality}

\begin{abstract}
Early Maladaptive Schemas (EMS) are patterns of developed emotional and cognitive responses of the interaction between personality and experiences in childhood. The purpose of this study was to investigate the relationships between EMSs and clinical dimensions of personality in a non-clinical sample. For this, 189 university students answered the sociodemographic questionnaire, instruments of anxious and depressive symptomatology, attachment and maladaptive schemas. Schema domains correlated with all clinical dimensions of personality. The data suggest that individuals with the dimensions Dependence, Mood Instability and Critical Avoidance are more likely to present Disconnection / Rejection and Autonomy and Impaired Performance schemas. The research allows the integration of the study of Personality and Schema Therapy.
\end{abstract}

Keywords: clinical dimensions of personality, early maladaptive schemas, cognitive vulnerability.

\section{Relación entre dimensiones de esquemas desconexión y rechazo y perjuicio en autonomía y desempeño y dimensiones clínicas del personalidade}

\section{Resumen}

Esquemas Desadaptativos Tempranos (EDTs) son patrones de respuestas emocionales y cognitivas desarrolladas la interacción entre la personalidad y de las experiencias en la infancia. El objetivo de este estudio fue investigar la relación entre los EDTs y dimensiones clínicas del personalidad en un muestra clínica. Para eso 189 estudiantes respondieron el "Cuestionario de esquemas de Young", Inventario de dimensiones clínicas del personalidade y los inventarios Beck de depresion y ansiedad. Las dimensiones del esquemas desadaptativos tempranos se correlacionaron con todas las dimensiones clínicas de personalidad. Los datos sugieren que los individuos con las dimensiones Dependencia, Inestabilidad del Humor y la Evitación de las Críticas son más propensos a presentar esquemas de la dimensión Desconexión y Rechazo y Perjuicio en Autonomía y Desempeño. El trabajo permite la integración del estudio de de la personalidad y de la terapia centrada em esquemas.

Palabras clave: dimensiones clínicas de la personalidad, esquemas desadaptativos tempranos, vulnerabilidad cognitiva. 
Young, Klosko e Weishaar (2009) ao abordarem o desenvolvimento da vulnerabilidade cognitiva como crenças que possuem componentes adaptativos, mas que se tornaram extremistas e rígidas ao longo da vida, afirmam que os indivíduos que experimentam repetidos eventos nocivos na infância são propensos a desenvolver traços disfuncionais chamados Esquemas Iniciais Desadaptativos (EIDs). Os EIDs são padrões emocionais e cognitivos rígidos que trazem prejuízos para o sujeito e possuem um conteúdo temático específico (Zeigler-Hill, Green, Arnau, Sisemore, \& Myers, 2011). Eles operam no nível mais profundo do processamento de informação e têm sido constantemente associados aos transtornos de personalidade (Barazandeh, Kissane, Saeedi, \& Gordon, 2016; Corral \& Calvete, 2014; Jacob \& Arntz, 2013). Essa perspectiva corrobora com a visão teórica de que crenças sobre si mesmo constituem uma variável importante para a compreensão do desenvolvimento e manutenção de transtornos psiquiátricos e podem ser um fator de vulnerabilidade individual, especialmente quando associadas a situações estressoras (Calvete, Orue \& Hankin, 2015).

Young et al. (2009) categorizou 18 EIDs que se encontram inseridos em cinco domínios. O primeiro domínio, desconexão/rejeição, relaciona-se a sentimentos de frustração de expectativas de segurança, estabilidade, carinho, empatia e aceitação. O segundo domínio, autonomia e desempenho prejudicados, abrange sentimentos de incapacidade ativados, falta de autonomia para sobrevivência de forma independente e crença na incapacidade de ter bom desempenho (Cazassa \& Oliveira, 2012). O terceiro domínio, limites prejudicados compreende a dificuldade de respeitar os direitos de terceiros, ajudar e realizar objetivos de longo prazo. O quarto domínio, direcionamento para o outro, diz respeito à prioridade em atender as necessidades dos outros em detrimento das suas próprias. O quinto domínio, supervigilância e inibição, engloba a supressão de sentimentos e impulsos para o cumprimento de regras.

A presença de esquemas dos domínios 1 e 2 é frequentemente notada em grupos clínicos, como os esquemas de abandono, desconfiança, privação emocional, defectividade, vulnerabilidade ao dano, dependência e fracasso no transtorno depressivo maior (Calvete, 2014), nos transtornos de ansiedade (Williams, Harfmann, Ingram, Hagan, \& Kramer, 2015), em pacientes suicidas (Méa, Zancanella, Ferreira, \& Wagner, 2015), no transtorno obsessivo compulsivo (Kim, Lee, \& Lee, 2014), no transtorno de personalidade borderline (Lawrence, Allen, \& Chanen, 2011) e no transtorno de personalidade evitativo (Carr
\& Francis, 2010). Os esquemas desses dois domínios possuem semelhanças com as crenças centrais abordadas na terapia cognitiva de Beck (1991), como o sentido de perda (por exemplo, privação emocional e abandono) e desesperança (por exemplo, fracasso e desconfiança) (Cámara \& Calvete, 2012).

Além da compreensão sobre as crenças centrais, Beck, Rush, Shaw e Emery (1979) fazem referência a tipos de personalidade, sociotrópico e autônomo, que são dimensões dominantes, cujos conteúdos específicos estão associados à necessidade de aceitação, companhia e relações (sociotrópico) ou metas e independência (autônomo) (Tobar, López, \& López, 2016). De modos diferentes esses tipos de personalidade se associam aos transtornos emocionais, sendo, por exemplo, os indivíduos autonômicos mais propensos a desenvolver um transtorno depressivo em decorrência de uma falha pessoal, enquanto os indivíduos sociotrópicos podem ficar deprimidos após a perda de um relacionamento (Knapp \& Beck, 2008).

Esses dois domínios também aparecem relacionados a sintomas clínicos de ansiedade e depressão. Por exemplo, o estudo de Shorey, Elmquist, Anderson e Stuart (2015) investigou a relação entre os EIDs e sintomas ansiosos e depressivos em sujeitos submetidos a tratamento para abuso de substâncias. Os autores verificaram que enquanto os esquemas do domínio 1 estavam associados aos sintomas depressivos, 0 domínio 2 estava associado aos sintomas ansiosos.

A personalidade pode se manifestar de maneira funcional ou patológica. Suas características estão presentes desde a infância e permanecem relativamente estáveis ao longo da vida (Widiger, 2011). Os traços podem ser definidos como a parte da personalidade que tende a permanecer constante (Pacheco \& Sisto, 2003) ou como padrões relativamente estáveis de comportamento, motivação, emoção e cognição (DeYoung, 2010). Eles funcionam como uma tendência para se comportar de determinada forma ao longo do tempo e em diferentes contextos, influenciando escolhas e preferências em situações diferentes (Malloy-Diniz, Fuentes, Mattos, \& Abreu, 2009).

Traços de personalidade podem ajudar a explicar a suscetibilidade individual ao estresse e aos transtornos psicológicos (Widiger, 2011) e são um fator de vulnerabilidade importante para a formação dos EIDs (Thimm, 2010). O modelo dimensional de psicopatologia segue a premissa de que todos os indivíduos possuem traços clínicos em algum nível, mas o agrupamento destes compõe as dimensões comuns aos transtornos de personalidade (Carvalho \& Primi, 2016). Identificar características de personalidade em grupos clínicos possibilita a intervenção nas tendências 
comportamentais, emocionais e cognitivas que trazem prejuízos às relações do indivíduo (Malloy-Diniz, Fuentes, Mattos, \& Abreu, 2009).

Bach, Markon, Simonsen e Krueger (2015) verificaram que as dimensões clínicas de personalidade podem ser expressas através das crenças disfuncionais e esquemas desadaptativos que terapeutas acessam no âmbito clínico. Considerando que EIDs e traços de personalidade possuem uma relação complementar (Lohr, Teglasi, \& French, 2004), estudos têm investigado as associações entre estes construtos. No estudo de Atalay, Akbas, Zahmacioglu e Kilic (2013) vários esquemas dos domínios 1 e 2 se correlacionaram de forma positiva com a dimensão de temperamento "evitação de danos" em uma amostra de estudantes universitários. O fator neuroticismo foi apontado como preditor da presença dos domínios de esquemas desconexão/rejeição, dependência/incompetência e direcionamento ao outro e como positivamente associado a todos os EIDs, com exceção de privação emocional, emaranhamento e inibição emocional (Soleymani \& Shahinejad, 2014; Thimm, 2010). O estudo de Carr e Francis (2010) verificou em uma amostra não clínica que o esquema de desconfiança/ abuso se correlacionou com traços de personalidade paranoide; isolamento social e inibição emocional com personalidade esquizoide; inibição emocional também se correlacionou com a personalidade evitativa, abandono com personalidade dependente e padrões inflexíveis com a personalidade obsessivo compulsiva.

O trabalho de Bach et al. (2015) em uma amostra clínica e não clínica comparou traços clínicos do Inventário de Personalidade do DSM-5 (Personality Inventory for DSM-5 - PID 5) com os EIDs. Eles verificaram que esquemas dos domínios 1 e 2 se sobrepõem aos traços clínicos de afetividade negativa e de distanciamento do Inventário de Transtorno de Personalidade do DSM-5, tendo um destaque os esquemas de abandono, inibição emocional e vulnerabilidade ao dano. Os autores afirmam que não é claro qual o fator primário da psicopatologia, os esquemas ou os traços, mas sugerem que estes últimos estão diretamente relacionados aos modos de ser, sentir e se comportar e podem ser mediados pelos esquemas desadaptativos.

Apesar do uso de populações clínicas em pesquisa apresentar vantagens, como a presença mais forte de sintomas e crenças (Cámara \& Calvete, 2012), esse tipo de amostra possui limitações, como a dificuldade em controlar comorbidades ou outras variáveis associadas aos transtornos que podem interferir nos resultados (Carr \& Francis, 2010). Pela perspectiva dimensional, os EIDs e as dimensões clínicas estão presentes em ambas as populações, clínicas e não clínicas, e variam ao longo de um continuum. Assim, é possível o uso de amostra não clínica para investigar dimensões psicopatológicas. Carr e Francis (2010) afirmam que amostras não clínicas podem ser até melhores em alguns casos, pois tendem a apresentar menor gravidade nos sintomas, não aumentam as correlações por variáveis intervenientes e representam mais claramente a prevalência de fatores etiológicos.

Sabendo que os esquemas dos domínios 1 e 2 estão presentes frequentemente em transtornos psiquiátricos e constituem um fator importante de vulnerabilidade clínica, identificar as dimensões clínicas de personalidade que predizem ou que estão mais associadas a estes domínios pode ajudar a identificar traços da personalidade importantes para o desenvolvimento de transtornos, auxiliando na formulação de caso e na identificação de alvo para tratamento, promovendo o caráter preventivo na intervenção. O objetivo deste trabalho é explorar as relações entre as dimensões clínicas de personalidade e os domínios 1 e 2 dos EIDs em amostra não clínica.

\section{Método}

\section{Aspectos éticos}

O estudo foi submetido e aprovado pelo Comitê de Ética em Pesquisa de Ensino Superior do Centro de Ciências da Saúde da Universidade Federal da Paraíba sob o número 1.198.116. Seguiram-se as diretrizes e normas regulamentadoras de pesquisas envolvendo seres humanos. Os participantes inicialmente receberam o Termo de Consentimento Livre e Esclarecido no qual consentiram a participação voluntária. Foram informados dos objetivos da pesquisa e a possibilidade de desistir a qualquer momento, sem nenhuma consequência negativa.

\section{Participantes}

Participaram da pesquisa 189 estudantes que cumpriram os critérios de inclusão do estudo. Eram universitários dos cursos de Administração (1,1\%), Agronomia (1,6\%), Arquitetura (3,2\%), Biblioteconomia (1,1\%), Ciências Contábeis $(15,9 \%)$, Direito (2,6\%), Enfermagem (2,1\%), Farmácia (1,6\%), Física (18,5\%), Fonoaudiologia (2,1\%), Medicina $(21,7 \%)$, Pedagogia $(3,7 \%)$, Psicologia $(21,1 \%)$ e Serviço Social $(3,7 \%)$, sendo 105 mulheres e 84 homens, com idades entre 18 e 30 anos e média de 22,89 anos $(\mathrm{DP}=4,1)$. Em relação à etnia, 51,9\% se autodeclararam brancos, $41,8 \%$ pardos e $6,3 \%$ negros. $86,8 \%$ dos participantes eram solteiros, $8,5 \%$ casados, $1,6 \%$ divorciados e $3,1 \%$ responderam "outro". 
Os critérios de exclusão foram: estar em tratamento psiquiátrico, fazer uso de medicação psicotrópica e/ou ter ideação suicida atual, informações verificadas através do questionário sociodemográfico. Foram incluídos neste estudo estudantes universitários que responderam aos questionários e não satisfaziam os critérios de exclusão.

\section{Materiais}

Questionário Sociodemógrafico. Instrumento voltado à coleta de informações gerais da amostra, como idade, sexo, religião, estado civil, escolaridade, renda familiar, se já fez ou faz tratamento psiquiátrico, fez ou faz psicoterapia, faz uso de medicação psicotrópica, e se há ideação suicida atual.

Inventário Dimensional Clínico da Personalidade -2 . Na perspectiva de avaliar aspectos clínicos da personalidade, Carvalho e Primi (no prelo) desenvolveram no Brasil o Inventário Dimensional Clínico da Personalidade (IDCP), composto por 12 dimensões: Dependência, Agressividade, Instabilidade de Humor, Excentricidade, Necessidade de Atenção, Desconfiança, Grandiosidade, Desapego, Evitação Social, Autossacrificio, Conscienciosidade e Impulsividade. O IDCP-2 possui 208 itens divididos em 12 dimensões. A classificação de cada item varia de 1 a 4 pontos que vão desde: Nada (não tem nada a ver comigo) a Muito (tem muito a ver comigo). Altas pontuações sugerem características tendenciosas para personalidade patológica (Carvalho, 2011). O IDCP-2 é uma versão adaptada do IDCP 1, que se encontra em processo de comercialização. O IDCP possui adequação nos índices de fidedignidade, com alfa de Cronbach superior a 0,7 para 11 dimensões completas do instrumento e evidências de validade em sua estrutura interna e em relação às variáveis externas, como as dimensões e facetas do Inventário de Personalidade NEO-PI Revisado (NEOPI-R) (Carvalho, 2011; Carvalho, Sette, \& Primi, 2014).

Inventários de Beck. O Inventário de Depressão de Beck (BDI) e o Inventário de Ansiedade Beck (BAI), desenvolvidos por Beck et al. (1996), foram utilizados para avaliar a sintomatologia ansiosa e depressiva, sendo utilizadas as versões disponíveis para pesquisa. Considerando a relação observada entre os EIDs dos domínios 1 e 2 e a sintomatologia ansiosa e depressiva (Shorey et al., 2015), incluiu-se esses instrumentos para verificar as relações entre essas variáveis em uma amostra não clínica. Ambas as escalas contêm 21 perguntas com classificações variando de 0 a 3 pontos cada. Assim, os escores podem variar de 0 a $63 \mathrm{com}$ pontuações mais altas indicando sintomatologia atual mais grave. As versões brasileiras dos inventários Beck apresentam boas propriedades psicométricas, com alfa de Cronbach de 0,79 e 0,83 e taxa de confiabilidade de 0,91 e 0,92 para o Inventário de Depressão de Beck (BDI) e o Inventário de Ansiedade Beck (BAI), respectivamente.

Questionário de Esquemas de Young. A avaliação dos Esquemas Iniciais Desadaptativos foi feita pelo Questionário de Esquemas de Young-forma reduzida (YSQ-S2), validado por Cazassa e Oliveira (2012) para a população brasileira apresentando índice de consistência interna total de $\alpha=0,95$, considerando os 75 itens (Cazassa \& Oliveira, 2012). O instrumento objetiva avaliar 15 Esquemas Iniciais Desadaptativos (EIDs) e possui uma escala tipo Likert de 1) Não me descreve de modo algum a 6) Me descreve perfeitamente - para pontuação de acordo com a percepção do examinando. Os 15 esquemas avaliados nesse instrumento são: fracasso, inibição emocional, privação emocional, autossacrifício, abandono, emaranhamento (self subdesenvolvido), autocontrole insuficiente, vulnerabilidade a danos ou a doenças, defectividade/vergonha, dependência/incompetência, padrões inflexíveis, subjugação, desconfiança/abuso, isolamento social e merecimento.

O questionário proporciona escores para cada esquema e o instrumento completo produz escores que variam de 75 a 450 pontos, quanto mais alto o escore maior a indicação de presença de EIDs. Para análise de correlação e regressão, somaram-se os escores dos fatores do YSQ-S2: abandono, isolamento social, privação emocional, defectividade e desconfiança (esquemas referente ao domínio desconexão/rejeição) para a variável domínio 1; e os escores dos fatores do YSQ-S2: fracasso, dependência, emaranhamento e vulnerabilidade a danos (esquemas referente ao domínio dependência/incompetência) para a variável domínio 2. Para a descrição da prevalência dos esquemas, foi utilizada a média dos escores médios dos esquemas dos domínios 1 e 2. A média dos escores do domínio 1, que possui cinco esquemas, pode variar entre 5 e 30 pontos; no domínio 2 , que possui quatro esquemas, a média dos escores médios pode variar entre 4 e 24 pontos. Assim, escores abaixo de 15 pontos para o domínio 1 e abaixo de 12 pontos para o domínio 2 indicam baixa prevalência de EIDs nestes domínios.

\section{Procedimentos}

Cada participante foi convidado a participar do estudo diretamente pelos pesquisadores envolvidos na coleta de dados. Com o objetivo de dirimir um possível efeito de cansaço ao responder os questionários, a ordem de apresentação dos instrumentos IDCP-2, BAI, BDI 
e YSQ-S2 foi randomizada, permanecendo constante apenas o Termo de Consentimento e o questionário de dados sociodemográficos ao início da coleta. A duração da aplicação foi, em média, 45 minutos.

\section{Análise de dados}

Os dados foram analisados através do programa Statistical Package for the Social Sciences (SPSS) versão 22.0. As análises descritivas consideradas foram as médias, desvios padrão e frequências. Foi utilizado o teste de correlação de Pearson para verificar as correlações entre os domínios 1 e 2 de esquemas e as 12 dimensões clínicas do IDCP-2 e os escores do BAI e BDI. Foi utilizado o teste Bonferroni para correção dos $p$-valores, utilizando-se como critério de significância o $p<0,001$. Utilizou-se a classificação da magnitude do efeito de correlação de Dancey e Reidy (2013), fraco: $\mathrm{r}=0,10$ até 0,30 ; moderado: $\mathrm{r}=0,40$ até 0,60 ; forte: $\mathrm{r}=0,70$ até 1 .

Em seguida, foram realizadas duas regressões múltiplas através do método stepwise, que é um método de explorar relações entre variáveis. Como este estudo é exploratório buscou-se investigar o poder preditivo das dimensões clínicas de personalidade sobre os domínios 1 e 2 de EIDs. Nesse tipo de regressão, a sequência de entrada dos preditores foi determinada por critérios estatísticos. Assim, as dimensões clínicas do IDCP-2 que apresentaram correlações significativas de maior magnitude com os domínios 1 e 2 foram adicionadas como variáveis preditoras de cada domínio de esquema. Este método permite a identificação de efeito de interação (mediação ou moderação) entre as variáveis.

Para a primeira regressão foi utilizado o domínio 1 como variável de desfecho e como variáveis preditoras entraram as seguintes dimensões: dependência, instabilidade de humor e evitação a críticas. $\mathrm{Na}$ segunda, utilizou-se o domínio 2 como variável de desfecho e como variáveis preditoras as dimensões: dependência, autossacrifício e evitação a críticas.

\section{Resultados}

\section{Descrição da amostra}

A média dos escores do BAI foi de 12,4 $(\mathrm{DP}=8,4)$ e do BDI foi de 9,59 $(\mathrm{DP}=6,8)$, indicando que os participantes desse estudo apresentam baixa sintomatologia ansiosa e depressiva. A média dos fatores para as dimensões clínicas de personalidade também foi baixa, variando de $1,5(\mathrm{DP}=0,45)$ para o fator impulsividade a $1,97(\mathrm{DP}=0,66)$ para o fator dependência. A média da soma dos escores dos esquemas no YSQ-S2 para o domínio 1 foi 10,26
$(\mathrm{DP}=3,89)$ e para o domínio 2 foi $8,0(\mathrm{DP}=3,15)$, indicando também a baixa presença de esquemas desadaptativos destes domínios na amostra.

\section{Análises inferenciais}

Observou-se correlações positivas e significativas moderadas e fortes entre os escores do YSQ-S2 dos domínios 1 e 2 e todas as dimensões clínicas de personalidade (Tabela 1). Para o domínio 1 as correlações de maior magnitude foram com as dimensões: dependência $(\rho=0,76, p<0,001)$, instabilidade de humor $(\rho=0,73, p<0,001)$ e evitação a críticas $(\rho=0,72, p<0,001)$. Para o domínio 2 as correlações de maior magnitude foram com as dimensões: dependência $(\rho=0,76, \quad p<0,001)$, autossacrifício $(\rho=0,69, p<0,001)$ e evitação a críticas $(\rho=0,69, p<0,001)$ (Tabela 1).

TABELA 1

Correlações entre os fatores do IDCP-2 e os fatores de esquemas dos domínios 1 e 2 do YSQ-S2

\begin{tabular}{lcc}
\hline \multicolumn{3}{c}{ Correlações - Valor de $\rho$} \\
\hline Domínio 1 & Domínio 2 \\
\hline Dependência &, $760^{* *}$ &, $760^{* *}$ \\
Agressividade &, $560^{* *}$ &, $454^{* *}$ \\
Instabilidade de Humor &, $734^{* *}$ &, $671^{* *}$ \\
Excentricidade &, $676^{* *}$ &, $538^{* *}$ \\
Necessidade de Atenção &, $550^{* *}$ &, $425^{* *}$ \\
Desconfiança &, $689^{* *}$ &, $511^{* *}$ \\
Grandiosidade &, $499^{* *}$ &, $330^{* *}$ \\
Isolamento &, $590^{* *}$ &, $466^{* *}$ \\
Evitação a Críticas &, $718^{* *}$ &, $693^{* *}$ \\
Autossacrifício &, $716^{* *}$ &, $695^{* *}$ \\
Conscienciosidade &, $492^{* *}$ &, $386^{* *}$ \\
Impulsividade &, $523^{* *}$ &, $411^{* *}$ \\
\hline
\end{tabular}

$* *: p<0,001$.

Os domínios 1 e 2 se correlacionaram forte e positivamente entre si $(\rho=0,80, p<0,001)$ e moderadamente com os escores do BAI e BDI. Para a sintomatologia ansiosa, o domínio 2 apresentou maior correlação $(\rho=0,57, p<0,001)$, seguido do domínio 1 $(\rho=0,53, p<0,001)$. Para a sintomatologia depressiva o domínio 1 apresentou maior correlação $(\rho=0,63$, $p<0,001)$ seguido do domínio $2(\rho=0,58, p<0,001)$.

Em seguida realizou-se duas regressões. A regressão com o domínio 1 como variável de desfecho foi realizada em 3 blocos com objetivo de identificar o efeito preditivo das variáveis nesta ordem: dependência, instabilidade de humor e evitação a críticas. A regressão com o domínio 2 como variável de 
desfecho foi realizada em três blocos com objetivo de identificar o efeito preditivo das variáveis dependência, evitação a críticas e autossacrifício, respectivamente.

Em conjunto, as variáveis selecionadas explicam $65,7 \%$ da variância do domínio 1 (desconexão/rejeição) de maneira significativa. Dependência é a variável que exerce maior influência sobre o domínio 1 explicando $58 \%$ da variância $\left(\mathrm{R}^{2}=0,58, \mathrm{~F}(1,177)=244,11\right.$, $p<0,001)$, seguida por instabilidade de humor, que explica 4,5\% $\left(\mathrm{R}^{2}=0,045, \mathrm{~F}(2,176)=146,47, p<0,001\right)$ e evitação a críticas, que explica $3,8 \%\left(\mathrm{R}^{2}=0,038\right.$, $\mathrm{F}(3,175)=114,54, p<001)$ (Tabela 2).

Para o domínio 2 (autonomia e desempenho prejudicados) as variáveis preditoras explicam $61,6 \%$, da variância e duas variáveis explicaram o modelo significativamente. A dimensão dependência explica $57,6 \%$ da variância $\left(\mathrm{R}^{2}=0,576, \mathrm{~F}(1,176)=239,35\right.$, $p<0,001)$, seguida por evitação a crítica, explicando $4,2 \%\left(\mathrm{R}^{2}=0,042, \mathrm{~F}(1,175)=141,53, p<0,001\right)$. A variável autossacrifício $\left(\mathrm{R}^{2}=0,004, \mathrm{~F}(3,174)=95,56, p=0,15\right)$ não prediz significativamente o domínio (Tabela 2).

A Tabela 3 apresenta os coeficientes das duas análises de regressão divididas em três etapas, permitindo identificar algumas interações. $\mathrm{Na}$ primeira regressão (domínio 1 ), no bloco 1 , a variável dependência apresenta coeficiente $\beta=0,76(t=15,62$, $p<0,001)$. Ao se adicionar a variável instabilidade de Humor $(\beta=0,36, t=4,59, p<0,001)$, no segundo bloco, observa-se uma redução significativa no coeficiente $\beta$ referente à variável dependência, que vai de 0,76 para $0,47(\mathrm{t}=5,93, p<0,001)$. Esse dado mostra uma interação entre as variáveis, que sugere uma ação mediadora da dimensão instabilidade de humor sobre dependência. Isto significa que parte do poder preditivo de dependência sobre os esquemas do domínio 1 parece ser mediado pela dimensão instabilidade de humor, e parte da variância da primeira variável é melhor explicada pela segunda. No terceiro bloco, a inclusão da variável evitação a críticas $(\beta=0,29, \mathrm{t}=4,43, p<0,001)$ acarreta uma pequena redução dos coeficientes $\beta$ das dimensões dependência $(\beta=0,34, \mathrm{t}=4,24, p<0,001) \mathrm{e}$ instabilidade de humor $(\beta=0,26, \mathrm{t}=3,29, p<0,001)$, sugerindo também uma pequena interação entre essas variáveis. Todas as variáveis permanecem predizendo significativamente o domínio.

Para a segunda análise de regressão, com o domínio 2 como variável de saída, no primeiro bloco, a variável dependência apresenta coeficiente $\beta=0,76$ $(\mathrm{t}=15,47, p<0,001)$. Adicionando a variável evitação a críticas $(\beta=0,30, \mathrm{t}=4,37, p<0,001)$, o coeficiente $\beta$ de dependência cai de 0,76 para $0,54(\mathrm{t}=7,81, p<0,001)$, indicando uma interação entre as variáveis, na qual

TABELA 2

Dados sumarizados da análise de regressão múltipla hierárquica para os domínios 1 e 2

\begin{tabular}{lcclcc}
\hline \multirow{2}{*}{ Variáveis Preditoras } & \multicolumn{2}{c}{ Domínio Desconexão/Rejeição } & \multirow{2}{*}{ Variáveis Preditoras } & & \multicolumn{2}{c}{ Autonomia e Desempenho Prejudicados } \\
\cline { 2 - 3 } & $R^{2}$ (Ajustado) & $R^{2}$ (Modificado) & & $R^{2}$ (Ajustado) & $R^{2}($ Modificado) \\
\hline 1 (Dependência) & 0,577 & $0,580^{* * *}$ & 1 (Dependência) & 0,574 & $0,576^{* * *}$ \\
2 (Instabilidade de Humor) & 0,620 & $0,045^{* * *}$ & 2 (Evitação a Crítica)s) & 0,614 & $0,042^{* * *}$ \\
3 (Evitação a Críticas) & 0,657 & $0,038^{* * *}$ & 3 (Autossacrifício) & 0,616 & 0,004 \\
\hline
\end{tabular}

$* * * p<0,001$.

TABELA 3

Coeficientes da análise de regressão múltipla hierárquica para os domínios 1 e 2

\begin{tabular}{|c|c|c|c|c|c|c|c|}
\hline \multirow{2}{*}{ Modelos } & \multicolumn{3}{|c|}{ Domínio Desconexão/Rejeição } & \multirow{2}{*}{ Modelos } & \multicolumn{3}{|c|}{ Autonomia e Desempenho Prejudicado } \\
\hline & $B$ & $E P B$ & $\beta$ & & $B$ & $E P B$ & $\beta$ \\
\hline Constante & 1,39 & 0,59 & & Constante & 0,77 & 0,49 & \\
\hline 1 Dependência & 4,48 & 0,28 & $0,76^{* * *}$ & 1 Dependência & 3,65 & 0,24 & $0,76^{* * *}$ \\
\hline Constante & 0,21 & 0,63 & & Constante & $-0,21$ & 0,51 & \\
\hline 2 Dependência & 0,75 & 0,46 & $0,47 * * *$ & 2 Dependência & 2,59 & 0,33 & $0,54 * * *$ \\
\hline Instabilidade de Humor & 2,44 & 0,53 & $0,36^{* * *}$ & Evitação a Críticas & 1,73 & 0,39 & $0,30 * * *$ \\
\hline Constante & 0,61 & 0,62 & & Constante & $-0,41$ & 0,53 & \\
\hline 3 Dependência & 2,00 & 0,47 & $0,34 * * *$ & 3 Dependência & 2,12 & 0,47 & $0,44 * * *$ \\
\hline Instabilidade de Humor & 1,74 & 0,53 & $0,26^{* * *}$ & Evitação a Críticas & 1,62 & 0,40 & $0,28 * * *$ \\
\hline Evitação a Críticas & 2,01 & 0,45 & $0,29 * * *$ & Autossacrifício & 0,69 & 0,49 & 0,13 \\
\hline
\end{tabular}

$* * * p<0,001$ 
evitação a críticas explica parte do efeito da dimensão dependência no domínio 2 . Adicionando-se a variável autossacrifício $(\beta=0,13 \mathrm{t}=1,41, \mathrm{p}=0,159)$, verifica-se que esta não explica significativamente a variável de saída e as variáveis preditivas dependência $(\beta=0,44$, $\mathrm{t}=4,50, \mathrm{p}<0,001)$ e evitação a críticas $(\beta=0,28, \mathrm{t}=4,05$, $\mathrm{p}<0,001)$ mantêm a significância.

\section{Discussão}

O objetivo deste estudo foi explorar as relações entre os domínios de EIDs desconexão/rejeição e autonomia e desempenho prejudicados e traços de personalidade clinicamente relevantes em uma amostra não clínica. Para tal utilizamos a versão curta do YSQ-S2 e o IDCP-2.

Neste estudo, a correlação entre os domínios 1 e 2 do questionário YSQ-S2 foi forte $(0,801, p<0,05)$, sugerindo a possibilidade da sobreposição entre eles. Estudos que avaliam a estrutura fatorial do YSQ apontam que os domínios autonomia e desempenho prejudicados e desconexão/rejeição estão bem estabelecidos (Calvete, Orue, \& González-Diez, 2013). Entretanto, o estudo de Saritaş e Gençöz (2011) propõe um modelo de compreensão do YSQ com três grandes fatores em vez de cinco. Em um desses três fatores estão os esquemas do domínio 1: privação emocional, desconfiança, defectividade, isolamento social e inibição emocional junto com fracasso que é um esquema tipicamente do domínio 2; enquanto que no segundo fator estão os esquemas do domínio 2: dependência, vulnerabilidade ao dano e emaranhamento associados a subjugação, autossacrifício e abandono, sendo este último esquema originalmente pertencente ao domínio 1 . O terceiro fator foi denominado limites prejudicados/padrões exagerados e foi composto pelos esquemas de grandiosidade, busca de aprovação, padrões rígidos, pessimismo e punição. Esse estudo é um exemplo que os domínios de esquemas estão associados e podem existir intersecções entre eles.

Os domínios 1 e 2 também se correlacionaram de forma moderada e positiva com os escores do BAI e BDI. Este dado corrobora com pesquisas que demonstram os esquemas destes domínios associados à sintomatologia ansiosa e depressiva (Dozois \& Rnic, 2015). O estudo de Calvete, Orue e Hankin (2015) investigou as influências de EIDs e estressores em sintomas depressivos e de ansiedade social em uma amostra de adolescentes. Os autores verificaram que esquemas do domínio desconexão/rejeição previram os sintomas depressivos e, os esquemas deste e dos domínios autonomia e desempenho prejudica- dos e direcionamento ao outro estão associados a maiores níveis de sintomas depressivos e ansiedade social. O estudo longitudinal de Braet, Vlierberghe, Vandevivere, Theuwis e Bosmans (2013) investigou os sintomas depressivos em adolescentes utilizando um conjunto de fatores do YSQ que chamaram de "esquema depressivo", composto por esquemas combinados dos domínios de desconexão/rejeição e autonomia e desempenho prejudicados: privação emocional, fracasso, defectividade/vergonha e dependência/incompetência. Os resultados desse estudo demonstraram que esses esquemas estavam claramente associados aos sintomas depressivos.

\section{EIDs e Dimensões Clínicas de Personalidade}

Os domínios 1 e 2 se correlacionaram positivamente com todas as dimensões clínicas de personalidade. A dimensão clínica dependência foi a variável com maior poder de predição tanto para o domínio 1 quanto para o 2 , sendo, porém, mediada por dimensões diferentes para cada domínio. Dependência representa o sentimento de ser menos capaz comparado às outras pessoas, temor de ser abandonado por quem gosta e posicionamento submisso, com necessidade de que os outros tomem decisões importantes para si (Carvalho, 2011). Sua definição apresenta semelhanças teóricas com os EIDs de dependência/incompetência (crença de que não se é capaz de lidar com as responsabilidades do dia a dia com competência e independência) do domínio autonomia e desempenho prejudicados e abandono (instabilidade percebida nos recursos disponíveis para o apoio ao indivíduo, cuidado emocional e proteção) do domínio desconexão/rejeição. Para Young et al. (2009) pacientes com os esquemas neste último domínio são incapazes de ter relações seguras e satisfatórias.

Para o domínio desempenho e autonomia prejudicados os sujeitos têm dificuldade em funcionar de forma independente (Young, Klosko, \& Weishaar, 2009), assim a dimensão de personalidade dependente pode influenciar o sujeito nas escolhas de experiências ao longo da vida e no reforçamento e manutenção de esquemas de incapacidade. A dimensão dependência também explica parte dos esquemas do domínio 1; o que faz sentido tendo em vista o temor de ser abandonado comum aos sujeitos com esquemas nesse domínio que tendem a apresentar relações conturbadas e medo de abandono (Young, Klosko, \& Weishaar, 2009). Este resultado sugere que a dimensão dependência pode ser um dos fatores associados a experiências difíceis na infância para o desenvolvimento de EIDs dos domínios 1 e 2 . 
Segundo o trabalho de Abela (2013) a dimensão dependência do IDCP está associada ao transtorno de personalidade dependente (Carvalho \& Pianowski, 2015). Schmidt (1994) em um estudo com amostra não clínica, também encontrou que o esquema de dependência/incompetência estava associado ao transtorno de personalidade dependente. O estudo de Reeves e Taylor (2007) investigou a relação entre EIDs e sintomas de transtornos de personalidade em uma amostra não clínica encontrando uma forte relação entre transtorno de personalidade dependente com o esquema de abandono. No estudo de Jovev e Jackson (2004) com pacientes diagnosticados com transtorno de personalidade dependente, os esquemas de abandono, defectividade e dependência estavam associados ao transtorno. Deste modo, é possível que ambos os esquemas estejam presentes na personalidade dependente e estes podem ser mediados pela dimensão de personalidade dependência. Considerando a proposta de Young et al. (2009) para a formação do EIDs, a partir da interação entre o ambiente e personalidade, a dimensão dependência explica mais de $50 \%$ da variância tanto para o domínio 1 quanto para o 2 .

É importante frisar que no estudo de Carvalho e Pianowski (2015) dependência apresentou correlações com o fator neuroticismo do Inventário de Personalidade NEO, sendo essa relação esperada considerando as características de ansiedade e depressão comuns à dimensão dependência (Carvalho \& Pianowski, 2015). $\mathrm{O}$ traço neuroticismo do NEO tem sido frequentemente associado aos EIDs. Por exemplo, no estudo de Muris (2006), com adolescentes não clínicos, neuroticismo foi um fator preditivo para os EIDs de defectividade, fracasso, dependência, vulnerabilidade a danos, emaranhamento, subjugação e autossacrifício. O estudo de Thimm (2010) investigou os cinco traços de personalidade do NEO e a relação com os EIDs em pacientes ambulatoriais adultos, e verificou que neuroticismo estava correlacionado com todos os EIDs. Ehsan (2011) encontrou em uma amostra de estudantes universitários que os esquemas de privação emocional, abandono, isolamento social e vulnerabilidade ao dano previram de forma independente uma parcela significativa do neuroticismo.

Existe uma semelhança conceitual entre neuroticismo e a dimensão instabilidade de humor. Essa dimensão diz respeito à oscilação no humor, com tendências para impulsividade, sentimento de culpa, descontrole, ansiedade e tristeza (Carvalho, 2011). O estudo de Carvalho e Sette (2015) encontrou correlações entre o fator neuroticismo do NEO-PI-R e a dimensão instabilidade de humor e aponta que além das semelhanças entre os elementos centrais desses fatores, ambos estão associados ao funcionamento do transtorno de personalidade borderline.

A variável instabilidade de humor previu o domínio desconexão/rejeição e apresentou interação com dependência, explicando também parte do efeito apresentado por esta última no domínio. Semelhante à dimensão instabilidade de humor, os esquemas do domínio desconexão/rejeição estão associados aos modos de funcionamento do transtorno borderline (Kellogg \& Young, 2006). Os modos criança irritada e vulnerável, no transtorno borderline, são marcados por necessidades emocionais não satisfeitas que, em geral, formam os esquemas de abandono, privação emocional e isolamento (Kellogg \& Young, 2006).

Um traço também semelhante à instabilidade de humor é a afetividade negativa, traço clínico proposto para avaliação pelo DSM-5. No estudo de Bach et al. (2015), esse traço foi associado a 14 EIDs e estava substancialmente envolvido com os modos criança irritada e vulnerável. Assim, a dimensão instabilidade de humor parece ser um traço de personalidade que favorece o desenvolvimento de esquemas no domínio 1, quando associados a experiências de negligência ou abuso.

A dimensão evitação a críticas apresentou poder preditivo sobre o domínio 1 e 2 e neste último domínio a dimensão explica parte do efeito de dependência. Evitação a críticas se refere à tendência a exibir preocupação com o futuro, constrangimento em situações sociais e dificuldade em estabelecer relações ou envolvimento emocional (Carvalho, 2011). O estudo de Abela, Carvalho, Cho e Yazigi (2015) com amostra de 105 pacientes ambulatoriais diagnosticados com pelo menos um transtorno de personalidade, verificou que essa evitação a críticas apresentou alta pontuação em todos os grupos de pacientes. Além disso, maior pontuação nessa dimensão estava associada a maiores comorbidades nos pacientes com transtorno de personalidade evitativo. Isso sugere que evitação a críticas é uma dimensão clínica importante nos transtornos de personalidade.

Em relação a sua associação com esquemas, a dimensão evitação a críticas possui semelhanças com o esquema vulnerabilidade ao dano, pertencente ao domínio 2 , no tocante à preocupação com o futuro e em relação à crença de que algo ruim vai acontecer. Além disso, pode estar associada ao esquema de defectividade com a crença de que será humilhado e criticado e de isolamento social, tendo em vista o constrangimento diante de situações sociais, sentimento de não fazer parte da comunidade e dificuldades em se relacionar com as pessoas. 
É possível que evitação a críticas possa estar associada à estratégia de enfrentamento "evitação" proposta por Young et al. (2009). Nesse estilo de enfrentamento, os sujeitos buscam evitar a todo custo a ativação dos esquemas que promovem sofrimento, e por fazer isso, acabam se isolando e apresentando ainda mais dificuldades nas relações sociais (Young, Klosko \& Weishaar, 2009). Além disso, essa dimensão possui semelhanças ao traço distanciamento do DSM5 , que se refere à tendência a ser afetivamente restrito, deprimido, a se isolar, evitar intimidade, suspeitar e ficar ansioso em relação aos outros. No estudo de Bach et al. (2015) esse traço de personalidade se correlacionou com doze EIDs e mais fortemente com o esquema de isolamento social, pertencente ao domínio desconexão/rejeição.

\section{Contribuições e limitações}

Os achados desse estudo permitem a integração do estudo entre Personalidade e Terapia dos Esquemas. Para Young et al. (2009) a formação dos esquemas depende do temperamento emocional que pode ser agrupado em dimensões (exemplo, ansiosa, obsessiva, passiva, irritada) e das experiências que podem favorecer a formação dos padrões rígidos e disfuncionais (abuso, negligência, etc.) (Weingartz, 2012).

Coletivamente os dados do presente estudo sugerem que as dimensões clínicas de personalidade dependência e evitação a críticas estão associadas aos domínios 1 e 2 de EIDs e, também, a dimensão instabilidade de humor está associada aos esquemas do domínio 1.Conforme verificado pelos autores, este estudo é pioneiro no Brasil em investigar as associações entre dimensões clínicas de personalidade e esquemas iniciais desadaptativos. O uso do IDCP possibilita a discussão na perspectiva dimensional dos traços relacionados ao funcionamento disfuncional.

$\mathrm{O}$ estudo possui limitações. Inicialmente utilizou-se um desenho transversal para examinar relação entre dimensões de personalidade e EIDs. Apesar dessas construções serem pensadas como traços relativamente estáveis ao longo da vida (Wang, Halvorsen, Eisemann, \& Waterloo, 2010), ressalta-se a importância de investigações longitudinais. Além disso, as estratégias comportamentais e os modos de esquemas são variáveis importantes que podem mediar a relação entre personalidade e esquemas, fazendo-se necessária a investigação desses construtos associados em pesquisas futuras.

É importante ressaltar que existe a possível interpretação de que os efeitos encontrados entre as dimensões clínicas e os esquemas simplesmente refletem a sobreposição entre as escalas (Carr \& Francis, 2010). Por exemplo, a relação entre dependência e o domínio autonomia e desempenho prejudicados pode refletir uma dificuldade geral do indivíduo em se sentir autônomo. Assim, as duas medidas podem representar formas distintas de obter informações sobre o mesmo construto.

Utilizamos as correlações e análise de regressão para estabelecer as relações entre esquemas e as dimensões clínicas. Entretanto, as conclusões do estudo diante do desenho da pesquisa e das análises utilizadas são limitadas. As análises de correlação e regressão são frágeis para estabelecer a suscetibilidade de esquemas devido à presença de traços clínicos de personalidade. Sugere-se o uso de outros procedimentos estatísticos para estudos futuros. Além disso, deve-se ter em mente que o estudo foi realizado em uma amostra não clínica, o que significa que a maioria dos sujeitos apresentam níveis baixos de EIDs, fazendo-se necessários estudos comparando grupos de sujeitos saudáveis com diferentes grupos clínicos.

\section{Referências}

Abela, R. K. (2013). Evidências de validade para o Inventário Dimensional Clínico da Personalidade (IDCP) com base nos perfis de participantes com diagnóstico psiquiátrico. Dissertação de Mestrado, Universidade Federal de São Paulo (UNIFESP), São Paulo. (DOI INEXISTENTE)

Abela, R. K., Carvalho, L. D. F., Cho, S. J. M., \& Yazigi, L. (2015). Validity evidences for the dimensional clinical personality inventory in outpatient psychiatric sample. Paidéia (Ribeirão Preto), 25(61), 221-228. doi:10.1590/198243272561201510

Atalay, H., Akbas, N. B., Zahmacioglu, O., \& Kilic, E. Z. (2013). Are early maladaptive schemas, temperament and character dimensions correlated? Journal of Psychiatry, 3(2), 206-213. doi:10.4236/ojpsych.2013.32018

Bach, B., Markon, K., Simonsen, E., \& Krueger, R. F. (2015). Clinical utility of the DSM-5 alternative model of personality disorders: Six cases from practice. Journal of Psychiatric Practice, 21(1), 3-25. doi:10.1097/01. pra.0000460618.02805.ef 
Barazandeh, H., Kissane, D. W., Saeedi, N., \& Gordon, M. (2016). A systematic review of the relationship between early maladaptive schemas and borderline personality disorder/traits. Personality and Individual Differences, 94, 130-139. doi:10.1016/j.paid.2016.01.021

Beck, A. T., Rush, A. J., Shaw, B. F., \& Emery, G. (1979). Cognitive therapy of depression. New York: Guilford Press. (DOI INEXISTENTE)

Beck, A. T. (1991). Cognitive therapy: A 30-year retrospective. American Psychologist, 46(4), 368-375. doi:10.1037/0003066X.46.4.368

Beck, A. T., Steer, R. A., Ball, R., \& Ranieri, W. F. (1996). Comparison of Beck Depression Inventories-IA and-II in psychiatric outpatients. Journal of Personality Assessment, 67(3), 588-597. doi:10.1207/s15327752jpa6703_13

Braet, C., Vlierberghe, L. V., Vandevivere, E., Theuwis, L., \& Bosmans, G. (2013). Depression in Early, middle and late adolescence: differential evidence for the cognitive diathesis-stress model. Clinical Psychology \& Psychotherapy, 20(5), 369-383. doi:10.1002/cpp.1789

Calvete, E. (2014). Emotional abuse as a predictor of early maladaptive schemas in adolescents: contributions to the development of depressive and social anxiety symptoms. Child Abuse \& Neglect, 38(4), 735-746. doi:10.1016/j. chiabu.2013.10.014

Calvete, E., Orue, I., \& González-Diez, Z. (2013). An examination of the structure and stability of early maladaptive schemas by means of the Young Schema Questionnaire-3. European Journal of Psychological Assessment, 29(1), 283-290. doi:10.1027/1015-5759/a000158

Calvete, E., Orue, I., \& Hankin, B. L. (2015). A longitudinal test of the vulnerability-stress model with early maladaptive schemas for depressive and social anxiety symptoms in adolescents. Journal of Psychopathology and Behavioral Assessment, 37(1), 85-99. doi:10.1007/s10862-014-9438-x

Cámara, M. \& Calvete, E. (2012). Early maladaptive schemas as moderators of the impact of stressful events on anxiety and depression in university students. Journal of Psychopathology and Behavioral Assessment, 34(1), 58-68. doi:0.1007/s10862-011-9261-6

Carr, S. N. \& Francis, A. J. (2010). Early maladaptive schemas and personality disorder symptoms: An examination in a non-clinical sample. Psychology and Psychotherapy: Theory, Research and Practice, 83(4), 333-349. doi:10.1348/147608309X481351

Carvalho, L. D. F. \& Pianowski, G. (2015). Revision of the dependency dimension of the Dimensional Clinical Personality Inventory. Paidéia, 25(60), 57-65. doi:10.1590/1982-43272560201508

Carvalho, L. F. \& Primi, R. (no prelo). Inventário Dimensional Clínico da Personalidade. Itatiba, SP, Brasil: Universidade São Francisco. (DOI INEXISTENTE)

Carvalho, L. D. F. \& Primi, R. (2016). Prototype matching of personality disorders with the Dimensional Clinical Personality Inventory. Psicologia: Teoria e Pesquisa, 32(2). doi:10.1590/0102-3772e322214

Carvalho, L. D. F. \& Sette, C. P. (2015). Review and verification of the psychometric properties of the mood instability dimension of the Dimensional Clinical Personality Inventory. Acta Colombiana de Psicología, 18(2), 115-127. doi:10.14718/ACP.2015.18.2.10

Carvalho, L. D. F., Sette, C. P., \& Primi, C. G. C. R. (2014). Propriedades psicométricas da versão revisada da dimensão necessidade de atenção do inventário dimensional clínico da personalidade. Temas em Psicologia, 22(1), 147-160. doi:10.9788/TP2014.1-12

Carvalho, L. F. (2011). Desenvolvimento e Verificação das Propriedades Psicométricas do Inventário Dimensional Clínico da Personalidade (Tese de doutorado). Programa de Pós-Graduação Stricto Sensu em Psicologia, Universidade São Francisco, Itatiba, SP, Brasil. (DOI INEXISTENTE)

Cazassa, J. M. \& Oliveira, M. (2012). Validação brasileira do questionário de esquemas de Young: forma breve. Estudos de Psicologia, 29(1), 23-31. doi:10.1590/S0103-166X2012000100003

Corral, C. \& Calvete, E. (2014). Early maladaptive schemas and personality disorder traits in perpetrators of intimate partner violence. The Spanish Journal of Psychology, 17, 1-10. doi:10.1017/sjp.2014.1

Dancey, C. P. \& Reidy, J. (2013). Estatística sem matemática para psicologia. Porto Alegre: Penso Editora. (DOI INEXISTENTE)

DeYoung, C. G. (2010). Personality neuroscience and the biology of traits. Social and Personality Psychology Compass, 4(12), 1165-1180. doi:10.1111/j.1751-9004.2010.00327.x

Dozois, D. J. \& Rnic, K. (2015). Core beliefs and self-schematic structure in depression. Current Opinion in Psychology, 4, 98-103. doi:10.1016/j.copsyc.2014.12.008

Ehsan, H. B. (2011). The Evaluation of Prediction Potential Neuroticism and Extraversion According to Early Maladaptive Schemas. Procedia-Social and Behavioral Sciences, 30, 524-529. doi:10.1016/j.sbspro.2011.10.102

Jacob, G. A. \& Arntz, A. (2013). Schema therapy for personality disorders-a review. International Journal of Cognitive Therapy, 6(2), 171-185. doi:10.1521/ijct.2013.6.2.171

Jovev, M. \& Jackson, H. J. (2004). Early maladaptive schemas in personality disordered individuals. Journal of Personality Disorders, 18(5), 467-478. doi:10.1521/pedi.18.5.467.51325

Kellogg, S. H. \& Young, J. E. (2006). Schema therapy for borderline personality disorder. Journal of Clinical Psychology, 62(4), 445-458. doi:10.1002/jclp.20240

Kim, J. E., Lee, S. W., \& Lee, S. J. (2014). Relationship between early maladaptive schemas and symptom dimensions in patients with obsessive-compulsive disorder. Psychiatry Research, 215(1), 134-140. doi:10.1016/j. psychres.2013.07.036 
Knapp, P. \& Beck, A. T. (2008). Fundamentos, modelos conceituais, aplicações e pesquisa da terapia cognitiva Cognitive therapy: foundations, conceptual models, applications and research. Revista Brasileira de Psiquiatria, 30(2), S54-S64. doi:10.1590/S151644462008000600002

Lawrence, K. A., Allen, J. S., \& Chanen, A. M. (2011). A study of maladaptive schemas and borderline personality disorder in young people. Cognitive Therapy and Research, 35(1), 30-39. doi:10.1007/s10608-009-9292-4

Lohr, L. B., Teglasi, H., \& French, M. (2004). Schemas and temperament as risk factors for emotional disability. Personality and Individual Differences, 36(7), 1637-1654. doi:10.1016/j.paid.2003.06.011

Malloy-Diniz, L. F., Fuentes, D., Mattos, P., \& Abreu, N. (2009). Avaliação Neuropsicológica. Porto Alegre, RS: Artmed Editora. (DOI INEXISTENTE)

Méa, C. P. D., Zancanella, S., Ferreira, V. R. T., \& Wagner, M. F. (2015). Early maladaptive schemas in hospitalized patients for suicide attempt. Revista Brasileira de Terapias Cognitivas, 11(1), 3-9. doi:10.5935/1808-5687.20150002

Muris, P. (2006). Maladaptive schemas in non-clinical adolescents: Relations to perceived parental rearing behaviours, big five personality factors and psychopathological symptoms. Clinical Psychology \& Psychotherapy, 13(6), 405-413. doi:10.1002/cpp.506

Pacheco, L. \& Sisto, F. F. (2003). Aprendizagem por interação e traços de personalidade. Psicologia Escolar e Educacional, 7(1), 69-76. doi:10.1590/S1413-85572003000100007

Reeves, M. \& Taylor, J. (2007). Specific relationships between core beliefs and personality disorder symptoms in a non-clinical sample. Clinical Psychology \& Psychotherapy, 14(2), 96-104. doi:10.1002/cpp.519

Saritaş, D. \& Gençö, T. (2011). Psychometric properties of" young schema questionnaire-short form 3" in a Turkish adolescent sample. Journal of Cognitive \& Behavioral Psychotherapies, 11(1), 83-96. (DOI INEXISTENTE)

Schmidt, N. B. (1994). The schema questionnaire and the schema avoidance questionnaire. Behaviour Therapist, 17, 90.

Shorey, R. C., Elmquist, J., Anderson, S., \& Stuart, G. L. (2015). The relationship between early maladaptive schemas, depression, and generalized anxiety among adults seeking residential treatment for substance use disorders. Journal of psychoactive drugs, 47(3), 230-238. doi:10.1080/02791072.2015.1050133

Soleymani, M. \& Shahinejad, P. (2014). Surveying the Correlation between Maladaptive Schemas, Personality Traits and Burnout among the Staff of Bank Mellat in West Azerbaijan Province. International Journal of Basic Sciences \& Applied Research, 3(11), 841-849. (DOI INEXISTENTE)

Thimm, J. C. (2010). Personality and early maladaptive schemas: A five-factor model perspective. Journal of Behavior Therapy and Experimental Psychiatry, 41(4), 373-380. doi:10.1016/j.jbtep.2010.03.009

Tobar, R. A. T., López, H. A. A., \& Sarmiento López, J. C. (2016). Sociotropía y autonomía:¿ evidencias para la hipótesis de especificidad de síntomas en depresión? Psicologia Clínica, 28(3), 35-52. (DOI INEXISTENTE)

Wang, C. E., Halvorsen, M., Eisemann, M., \& Waterloo, K. (2010). Stability of dysfunctional attitudes and early maladaptive schemas: a 9-year follow-up study of clinically depressed subjects. Journal of Behavior Therapy and Experimental Psychiatry, 41(4), 389-396. doi:10.1016/j.jbtep.2010.04.002

Weingartz, D. P. S. J. P. (2012). Zur Gültigkeit des Schemamodells nach Jeffrey Young in Deutschsprachigen Stichproben (Doctoral dissertation). Fachbereichs Humanwissenschaften, Universität Osnabrück, Osnabrück. (DOI INEXISTENTE)

Widiger, T. A. (2011). Personality and psychopathology. World Psychiatry, 10(2), 103-106. doi:10.1002/j.2051-5545.2011. tb00024.x

Williams, C. L., Harfmann, E. J., Ingram, R. E., Hagan, K. E., \& Kramer, N. M. (2015). Specificity of parental bonding and rumination in depressive and anxious emotional distress. Personality and Individual Differences, 79, 157-161. doi:10.1016/j.paid.2015.01.055

Young, J. E., Klosko, J. S., \& Weishaar, M. E. (2009). Terapia do Esquema: Guia de Técnicas Cognitivo-Comportamentais Inovadoras. Porto Alegre, RS: Artmed Editora. (DOI INEXISTENTE)

Zeigler-Hill, V., Green, B. A., Arnau, R. C., Sisemore, T. B., \& Myers, E. M. (2011). Trouble ahead, trouble behind: Narcissism and early maladaptive schemas. Journal of Behavior Therapy and Experimental Psychiatry, 42(1), 96-103. doi:10.1016/j.jbtep.2010.07.004

Dados dos autores:

Natany de Souza Batista Medeiros - Mestre, Universidade Federal do Rio Grande do Norte.

Bruno Gonçalves de Medeiros - Psicólogo, Universidade Federal do Rio Grande do Norte.

Victor Hugo Dias Pereira - Psicólogo, Universidade Federal da Paraíba.

Ismael Ferreira da Costa - Mestre, Universidade Federal da Paraíba.

Melyssa Kellyane Cavalcanti Galdino - Doutora, Universidade Federal da Paraíba.

Endereço para correspondência:

Natany de Souza Batista Medeiros

Av. Nilo Peçanha 620, Petrópolis - 3ำ subsolo do HUOL

59012-300, Natal, RN, Brasil

E-mail: natanypsicologia@gmail.com

Recebido em: 06/07/2017.

Aceito em: 10/09/2018.

Publicado em: 09/05/2019. 